\title{
Caractérisation du fruit du baobab et étude de sa transformation en nectar
}

\author{
Mady CISSE ${ }^{1}$, Mama SAKHO ${ }^{1}$, Manuel DORNIER ${ }^{2 \star}$ Codou MAR DIOP $^{1}$, Max REYNES ${ }^{2}$, Oumar SOCK ${ }^{1}$
}

${ }^{1}$ ESP, Univ. Cheikh Anta Diop, BP 5085, Dakar Fann, Sénégal

2 UMR 95 Qualisud, Cirad, Montpellier SupAgro, 73 rue Jean-François Breton,

TA B-95 / 16,

34398 Montpellier Cedex 5 ,

France

dornier@cirad.fr

\section{* Correspondance et tirés à part}

Reçu le 4 juin 2008

Accepté le 8 août 2008

Fruits, 2009, vol. 64, p. 19-34 (C) 2009 Cirad/EDP Sciences All rights reserved DOI: $10.1051 /$ fruits:2008052 www.fruits-journal.org RESUMEN ESPAÑOL, p. 34

\section{Characterization of the baobab tree fruit and study of its processing into nectar.}

Abstract - Introduction. The baobab tree (Adansonia digitata L.) grows wild in Senegal where the fruit is consumed in various forms. Although the plant ecology and botany have been well studied, works relating to the fruit processing or the impact of processing on the quality of the final products are rare. In order to evaluate the technological potential of these fruits, as a precondition to the development of their processed products on the local, regional and international markets, we attempted to characterize the raw material better (biochemical composition and nutritional quality), and we studied the processing of the product into nectar. Materials and methods. Our study related to twelve without-shell fruit samples of A. digitata collected between 2004 and 2006 in various zones of Senegal. Proportions of the various components of the fruit (pulp, seeds, fibers) were assessed, then various physico-chemical characteristics of the pulp and seed were analyzed. The microbial flora present in traditionally prepared drinks was studied. Pasteurization techniques and crossflow microfiltration of nectars were applied. Various uses and traditional fruit processing were evaluated starting from a survey carried out with a sample of 93 people questioned in Dakar and Saly. Results and discussion. The pulp was characterized by a low water content (6-15\%), a strong acidity (68$201 \mathrm{mEq} \cdot 100 \mathrm{~g}^{-1}$ ) and high contents of sugars (20-32\%), ascorbic acid (125-312 mg $\cdot 100 \mathrm{~g}^{-1}$ ), minerals (6\%) and starch (41-47\%). The chromatograms obtained by GC/MS on the fruit pulp identified 10 aroma compounds, especially isopropyl myristate, an unidentified phenolic compound and nonanal. Without special precaution, the pulp composition evolved during storage and its quality was quickly degraded. The survey carried out showed that the fruit pulp of the baobab tree is mainly used for the development of drinks of nectar type with a [fruit / water] mass ratio of [1 / 3]. Stabilization of nectar can be obtained by pasteurization $\left(80^{\circ} \mathrm{C} / 10 \mathrm{~min}\right)$. Nevertheless, heat treatment damaged the product quality. When cold stabilization by crossflow microfiltration was carried out on nectars with [fruit / water] mass ratios of [1/3] and [1/5], the permeate flux was between $\left[(23\right.$ and 77$\left.) \mathrm{L} \cdot \mathrm{h}^{-1} \cdot \mathrm{m}^{-2}\right]$ for a volumetric reduction ratio ranging between 1 and 2 . These low permeate fluxes compromise the potential of crossflow microfiltration for the stabilization of the nectar. Conclusion. In the future, from the point of view of a local production of nectar from the baobab tree fruit, research will have to be focused on the thermal stabilization of drinks.

\section{Senegal / Adansonia / fruit juices / keeping quality / pasteurizing / microfiltration}

\section{Caractérisation du fruit du baobab et étude de sa transformation en nectar.}

Résumé - Introduction. Le baobab (Adansonia digitata L.) est un arbre qui pousse à l'état sauvage au Sénégal où le fruit est consommé sous différentes formes. Si l'écologie et la botanique de la plante ont été bien étudiées, les travaux portant sur la transformation du fruit ou sur l'impact des traitements de transformation sur la qualité des produits finis sont rares. Afin d'évaluer le potentiel technologique de ces fruits, en préalable au développement de leurs produits de transformation sur les marchés locaux, régionaux et internationaux, nous avons cherché à mieux caractériser la matière première (composition biochimique et qualité nutritionnelle), et nous avons étudié la transformation du produit en nectar. Matériel et méthodes. L'étude a porté sur douze échantillons de fruits décoqués d'A. digitata prélevés entre 2004 et 2006 dans différentes zones du Sénégal. Les proportions des différents constituants du fruit (pulpe, graines, fibres) ont été déterminées, puis différentes caractéristiques physicochimiques de la pulpe et de la graine ont été analysées. La flore microbienne présente dans les boissons traditionnellement préparées a été étudiée. Les techniques de pasteurisation et de microfiltration tangentielle des nectars ont été appliquées. Les différentes utilisations et les procédés de transformation traditionnels du fruit ont été évalués à partir d'une enquête réalisée sur un échantillon de 93 personnes interrogées à Dakar et Saly. Résultats et discussion. La pulpe a été caractérisée par une faible teneur en eau (6-15\%), une forte acidité (68-201 mEq·100 g ${ }^{-1}$ ) et des teneurs élevées en sucres (20-32\%), acide ascorbique (125-312 mg·100 $\mathrm{g}^{-1}$ ), minéraux (6\%) et amidon (41-47\%). Les chromatogrammes obtenus par CPG/SM sur la pulpe de fruit ont mis en évidence 10 composés d'arôme dont le myristate d'isopropyle, un composé phénolique non identifié et le nonanal. Sans précaution particulière, la composition de la pulpe a évolué au cours du stockage et sa qualité s'est rapidement dégradée. L'enquête réalisée a montré que la pulpe du fruit de baobab est principalement utilisée pour l'élaboration de boissons de type nectar avec un rapport massique [fruit / eau] de [1 / 3]. La stabilisation du nectar peut être obtenue par pasteurisation $\left(80^{\circ} \mathrm{C} / 10 \mathrm{~min}\right)$ au détriment de la qualité du produit. Des essais de stabilisation à froid par microfiltration tangentielle ont été réalisés sur des nectars avec des rapports massiques [fruit / eau] de [1/3] et [1/5]. Les densités de flux ont été comprises entre [(23 et 77$\left.) \mathrm{L}^{-1} \mathrm{~h}^{-1} \cdot \mathrm{m}^{-2}\right]$ pour des facteurs de réduction volumique compris entre 1 et 2 . Les faibles densités de flux de perméat mesurées compromettent l'intérêt de la microfiltration tangentielle pour la stabilisation à froid du nectar. Conclusion. À l'avenir, dans la perspective d'une production locale de nectar de fruit du baobab, les travaux de recherche devront être focalisés sur la stabilisation thermique de la boisson.

Sénégal / Adansonia / jus de fruits / aptitude à la conservation / pasteurisation / microfiltration 


\section{Introduction}

Le baobab (Adansonia digitata L.) est un arbre qui pousse à l'état sauvage au Sénégal $[1,2]$. On le rencontre également dans toutes les régions semi-arides et subhumides de l'Afrique intertropicale, ainsi qu'à Madagascar [3, 4]. Le fruit du baobab ou pain de singe, appelé "buy " au Sénégal, est largement consommé sous différentes formes [58]. Les travaux scientifiques réalisés à ce jour sur l'espèce portent essentiellement sur l'étude de son écologie et de ses caractéristiques botaniques. La caractérisation biochimique du fruit est incomplète et les résultats sont disparates. Les références qui portent sur la transformation du fruit ou sur l'impact des traitements de transformation sur la qualité des produits finis sont rares [9, 10].

Le fruit du baobab reste sous-exploité régionalement malgré des caractéristiques nutritionnelles intéressantes. Il s'agit en effet d'un fruit réputé riche en fibres alimentaires, en minéraux et en vitamine $C[11,12]$. Au Sénégal, le fruit est exclusivement valorisé de manière traditionnelle sous forme de boissons destinées au marché de proximité. Ces produits, qui, pour la plupart d'entre eux, ne se conservent que quelques jours, sont très recherchés par la population locale pour leurs caractéristiques organoleptiques. L'évaluation du potentiel technologique de ces fruits est une première étape indispensable au développement de leurs produits de transformation sur les marchés locaux et régionaux, voire sur les marchés d'exportation.

Dans ce contexte, notre travail a visé tout d'abord à mieux caractériser la matière première en termes de composition biochimi-

Figure 1.

Zones géographiques d'origine des différents lots de fruits de baobab collectés au Sénégal pour en caractériser la pulpe et le nectar.

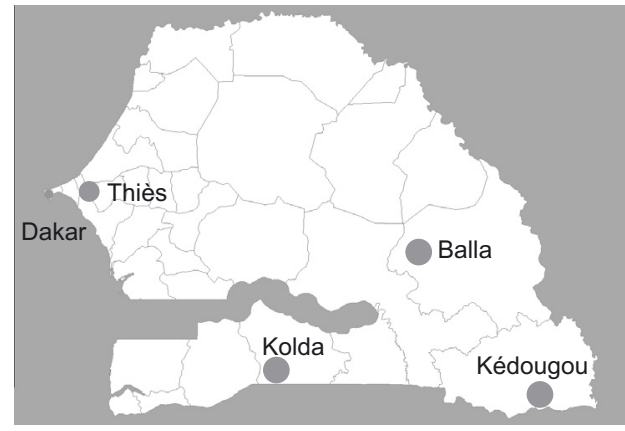

Les proportions des différents constituants du fruit (pulpe, graines, fibres) ont été déterminées par pesée, à $0,1 \mathrm{~g}$ près, uniquement sur trois échantillons : Balla 2004, Kédougou 2004 et Thiès 2004. Pour chaque échantillon, les mesures de masses ont été réalisées en triplicata sur environ $1 \mathrm{~kg}$ de fruit après séparation des constituants par un traitement mécanique modéré au mortier et pilon. La masse de coque par fruit n'a pas pu être évaluée au cours de ce travail qui n'a pas été conduit sur des fruits entiers mais 
sur des fruits décoqués ; à titre indicatif, la coque représente généralement entre (60 et 65) \% de la masse du fruit.

L'humidité, l'extrait sec soluble, le pH et l'acidité titrable, ainsi que les teneurs en cendres, amidon, sucres totaux et réducteurs, protéines et lipides ont été déterminés en suivant des méthodes d'analyse classiques [13]. La vitamine $\mathrm{C}$ a été dosée par oxydoréduction avec le dichloro-2, 6-phénolindophénol (2,6-DCPIP) après extraction à l'acide métaphosphorique et stabilisation à l'acide oxalique. L'éthanol a été mesuré par oxydation avec un excès de dichromate de potassium après distillation [14]. Les saccharose, glucose et fructose ont été déterminés à l'aide d'un kit enzymatique par spectrométrie UV (Boerhinger, Mannheim, Allemagne). La viscosité des solutions a été mesurée par un viscosimètre Searle à cylindres coaxiaux (Haake, VT 550). La mesure a été effectuée à diverses vitesses de cisaillement avec le module NV. Les polyphénols totaux ont été déterminés en suivant la méthode décrite par Georgé et al. [15], tandis que le pouvoir antioxydant a été évalué par la méthode ORAC (Oxygen Radical Absorbance Capacity) [16]. Les éléments minéraux ont été dosés par spectrophotométrie d'émission atomique par plasma à couplage inductif (ICP). Les composés d'arôme ont été analysés par SPME/CPG/SM dans les conditions décrites par Brat et al. [17]. Afin d'évaluer la répétabilité des mesures, une partie des analyses a été réalisée trois fois.

Les milieux PCA (Plate Count Agar), MRS (De Man, Rogosa, Sharpe) et DCL (Désoxycholate Citrate Lactose) ont été utilisés (Bio$\mathrm{Rad}, \mathrm{Ca}, \mathrm{USA}$ ), respectivement, pour évaluer la flore mésophile aérobie totale, les bactéries lactiques et les coliformes totaux. Le dénombrement a été effectué après $48 \mathrm{~h}$ d'incubation à $37^{\circ} \mathrm{C}$ des boissons préparées. Les levures et moisissures ont été recherchées sur milieu Sabouraud auquel du chloramphénicol à $0,05 \%$ a été ajouté et incubé à $30^{\circ} \mathrm{C}$ pendant $5 \mathrm{j}$. Les salmonelles ont été dénombrées après 5 j à $37{ }^{\circ} \mathrm{C}$ sur milieu SS (Salmonella Shigella) [18].

Un jury de dégustation constitué de 10 personnes de nationalité sénégalaise et habituées à la consommation de ce type de boisson a évalué la qualité organoleptique des nectars. Sans avoir recours à un entraînement préalable, des tests de notations ont été mis en place en utilisant six descripteurs : odeur, acidité, couleur, goût sucré, pulposité et homogénéité de la texture en bouche. Chaque membre du jury a évalué ces descripteurs sur une échelle qualitative de 1 à 9 , et a donné une appréciation individuelle globale sur les produits.

\subsection{Pasteurisation des nectars}

La pasteurisation des nectars a été réalisée par immersion dans un bain-marie chauffé après conditionnement à froid en bouteilles de verre de $250 \mathrm{~mL}$ munies de capsules twist-off. Avant utilisation, les bouteilles et les capsules ont été lavées puis désinfectées à l'hypochlorite de sodium à $100 \mathrm{mg} \cdot \mathrm{L}^{-1} \mathrm{de}$ chlore actif pendant 30 min à une température de $30{ }^{\circ} \mathrm{C}$. La température du produit a été suivie au cours du temps au centre d'une bouteille témoin à l'aide d'un thermomètre (Checktemp 1, Hanna instruments) possédant une gamme de lecture comprise entre $-50{ }^{\circ} \mathrm{C}$ et $+150{ }^{\circ} \mathrm{C}$ avec une précision de $\pm 0,2^{\circ} \mathrm{C}$ (échantillonnage toutes les minutes). Après l'application d'un barème préalablement choisi, les bouteilles ont été refroidies sous courant d'eau froide. Pour chaque traitement, la valeur pasteurisatrice totale subie par le produit a été calculée en prenant une température de référence de $70{ }^{\circ} \mathrm{C}$ et un facteur $z$ de $10^{\circ} \mathrm{C}$. Trois barèmes ont été utilisés pour obtenir des valeurs pasteurisatrices différentes : $70{ }^{\circ} \mathrm{C}$ pendant $10 \mathrm{~min}, 80^{\circ} \mathrm{C}$ pendant $10 \mathrm{~min}$ et $90^{\circ} \mathrm{C}$ pendant $5 \mathrm{~min}$.

\subsection{Microfiltration tangentielle des nectars}

Deux installations ont été mises en ouvre au cours de cette étude : un pilote semi-industriel IMECA (Clermont-l'Hérault, France) à boucle de circulation muni d'une membrane tubulaire multicanaux de $0,2 \mathrm{~m}^{2}$ (Membralox 1P19-4, Pall-Exekia, France) et un pilote de laboratoire TIA (Bollène, France) sans pompe de circulation muni de quatre membranes mono-tubulaires de $55 \mathrm{~cm}^{2}$ montées en série (Membralox). Toutes les membranes utilisées présentent un diamètre moyen de pores de 0,2 $\mu \mathrm{m}$. Chacune de ces instal- 
lations permet de contrôler les conditions opératoires : vitesse tangentielle $\left(2,0 \mathrm{~m} \cdot \mathrm{s}^{-1}<\right.$ $\left.\mathrm{U}<6,0 \mathrm{~m} \cdot \mathrm{s}^{-1}\right)$, température $\left(25^{\circ} \mathrm{C}<\mathrm{T}<41{ }^{\circ} \mathrm{C}\right)$ et pression transmembranaire $(1,00$ bar $<$ Ptm $<3,75$ bar). Selon les cas, les essais ont été réalisés avec extraction continue de perméat (facteur de réduction volumique FRV $>1)$ ou en recyclage total $($ FRV $=1)$.

\section{5. Études par voie d'enquêtes}

Afin d'identifier les différentes utilisations et de définir les procédés de transformation traditionnels du fruit, une enquête a été réalisée sur un échantillon de 93 personnes (42 ménagères, 27 vendeuses de fruits, 18 restauratrices et 6 restaurateurs) dans la zone urbaine de Dakar et à Saly $(80 \mathrm{~km}$ au sud de Dakar). L'enquête a été basée sur l'utilisation d'un questionnaire ouvert abordant les diverses utilisations du fruit et les procédures traditionnelles de transformation.

\section{Résultats et discussion}

\subsection{Proportions des constituants du fruit}

Les résultats de la mesure des bilans massiques (figure 2) ont montré que l'échantillon en provenance de Kédougou était le plus riche en pulpe (24\%), suivi par ceux de Balla (22\%) et de Thiès (15\%). L'échantillon en provenance de Balla a présenté le pourcentage en fibres le plus faible ( $4 \%$ )

\section{Figure 2.}

Pourcentages moyens des différents constituants du fruit du baobab collecté dans trois régions du Sénégal en 2004 (calcul fait sur trois échantillons de $1 \mathrm{~kg}$ pour chaque origine).

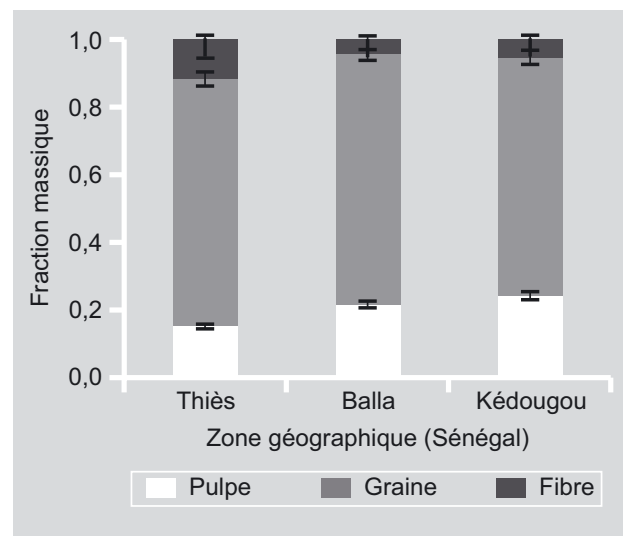

alors que celui de Thiès a été le plus riche (12\%). Ces différences pourraient être expliquées par divers facteurs, notamment par différentes conditions pédoclimatiques. En effet, les échantillons provenant du sud du pays (Kédougou et Balla), où les pluviométries sont plus importantes qu'à Thies, ont les pourcentages de pulpe les plus importants. Enfin, pour les trois échantillons de fruits, les graines ont représenté plus de $70 \%$ du poids total. Ces résultats concordent avec ceux obtenus par Soloviev et al. [12] et de Murray et al. [19].

\subsection{Composition du fruit}

\subsubsection{Principales caractéristiques de la pulpe}

L'analyse des caractéristiques mesurées sur les échantillons prélevés a montré que la teneur en eau de la pulpe était comprise entre ( 6 et 15$) \%$ (tableau I). Compte tenu de cette faible humidité, les teneurs en glucides se révèlent très élevées : de (41 à 48) \% d'amidon et de (22 à 32) \% de sucres totaux. Les sucres réducteurs représentent entre le quart et la moitié des sucres totaux.

Pour la plupart des échantillons analysés, l'acidité titrable de la pulpe a été très élevée [(68 à 201) $\mathrm{mEq} \cdot 100 \mathrm{~g}^{-1}$ ]. Son pH s'est révélé voisin de 3,3. Par ailleurs, une quantité importante d'acide ascorbique [(125 à 312) $\mathrm{mg} \cdot 100 \mathrm{~g}^{-1}$ ] trouvée dans cette pulpe permet de classer le produit parmi les fruits les plus riches en vitamine $\mathrm{C}$ après le camucamu (Myrciaria dubia (H.B.K.) Mc. Vaugh), l'acérole (Malpighia glabra L.), le ditax (Detarium senegalense Gmel.) ou le cassis (Ribes nigrum L.) [20, 21]. La teneur en matière grasse a oscillé entre $(0,2$ et 0,8$) \%$ et le taux de protéines a été environ de $2 \%$.

Parmi l'ensemble des caractéristiques étudiées, celles concernant l'acidité et la teneur en acide ascorbique de la pulpe du fruit de baobab ont montré la plus grande variabilité (coefficients de variation calculés sur les 12 échantillons $\geq 30 \%$ ). L'incidence des conditions pédoclimatiques, du stade de maturité du fruit à la récolte et des conditions de stockage de la pulpe sont autant de facteurs pouvant expliquer ces variations. Afin d'obtenir une teneur en vitamine $\mathrm{C}$ la 
Transformation du fruit du baobab

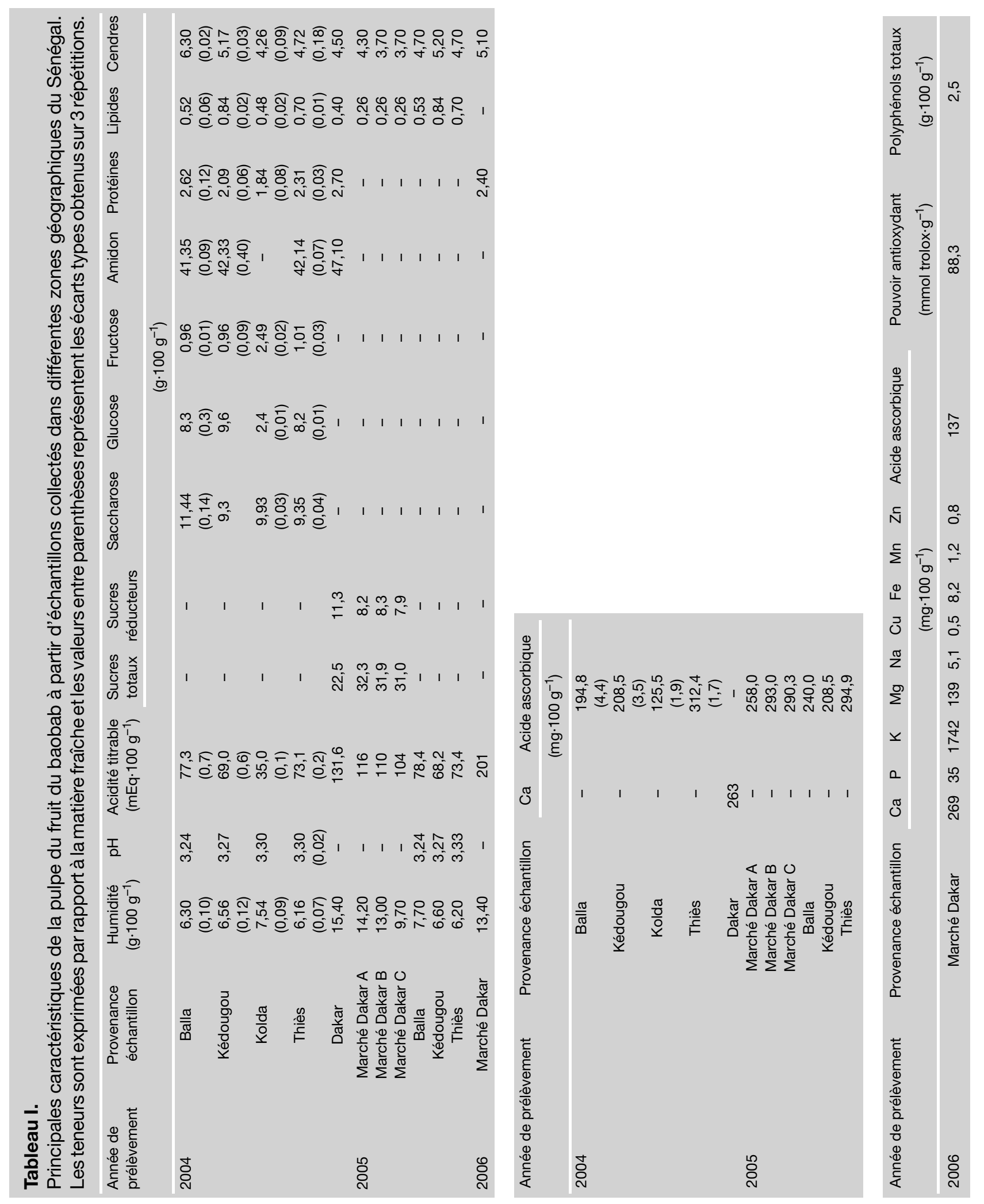


plus élevée possible, il sera nécessaire d'identifier les facteurs prédominants de variation.

La teneur en minéraux de la pulpe a été comprise entre $3,7 \%$ et $6,3 \%$, ce qui est remarquable pour un fruit. Comme dans la plupart des produits végétaux, le composé minéral qui a été prédominant a été le potassium. Les teneurs en calcium et magnésium ont également été assez intéressantes.

Évalué par la méthode ORAC sur un échantillon, le pouvoir anti-oxydant de la pulpe du fruit s'est révélé important $\left(88 \mu \mathrm{mol}\right.$ trolox $\left.\cdot \mathrm{g}^{-1}\right)$. Il est comparable à celui de la lentille (Lens culinaris Medik.) $\left(81 \mu \mathrm{mol}\right.$ trolox $\left.\cdot \mathrm{g}^{-1}\right)$, du raisin (Vitis vinifera L.) (87 $\mu$ mol trolox $\left.\cdot \mathrm{g}^{-1}\right)$, de la mûre (Rubus sp.) (72 $\mu \mathrm{mol}$ trolox $\cdot \mathrm{g}^{-1}$ ) ou de la tomate $\left(67 \mu \mathrm{mol}\right.$ trolox $\left.\cdot \mathrm{g}^{-1}\right)$, produits qui comptent parmi les plus riches en composés antioxydants $[22,23]$. Ce résultat est probablement lié à la teneur élevée de la pulpe en acide ascorbique et en composés phénoliques (> $2 \%)$. Il confère au fruit un intérêt nutritionnel supplémentaire.

Les chromatogrammes obtenus par CPG/ SM sur la pulpe de fruit ont permis de mettre en évidence dix composés d'arôme qui sont, dans l'ordre décroissant des aires de pics, le myristate d'isopropyle, un composé phénolique non identifié, le nonanal, le safranal, la géranone, le décanal, l'octanal, le linalol, un second composé phénolique et un composé sesquiterpénique non identifiés. Une étude plus approfondie sera néanmoins nécessaire pour confirmer la composition aromatique du produit et obtenir des données quantitatives.

\section{Figure 3.}

Évolution de la vitamine $C$ et de I'humidité de la pulpe de fruit du baobab au cours du stockage à température ambiante $\left(30^{\circ} \mathrm{C}\right)$. Mesures réalisées sur $300 \mathrm{~g}$ de l'échantillon prélevés à Kolda (Sénégal) en 2004.

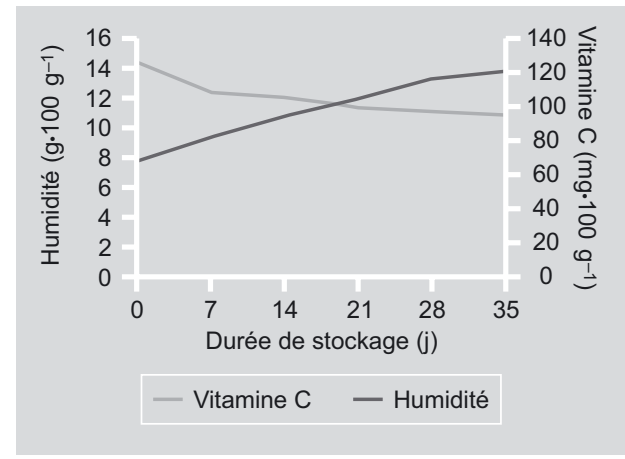

\subsection{2. Évolution de la pulpe au cours du stockage}

Afin d'évaluer la stabilité de la pulpe brute et l'incidence du stockage sur sa qualité, l'évolution de la teneur en eau, de l'acidité titrable et de la teneur en acide ascorbique a été suivie au cours d'un stockage de 35 j à température ambiante $\left(30^{\circ} \mathrm{C}\right)$ et à l'abri de la lumière (conditionnement en poches plastique non-étanches).

Les résultats ont montré que, en 1 mois de stockage, la teneur en eau de la pulpe augmentait fortement de plus de $80 \%$ (figure 3), ce qui mettrait en évidence le caractère hygroscopique de cette partie du fruit. Durant ce stockage, la teneur en vitamine $\mathrm{C}$, exprimée par rapport à la matière sèche, est passée de (126 à 94) $\mathrm{mg} \cdot 100 \mathrm{~g}^{-1}$. Cette diminution de $25 \%$ résulte probablement d'une dégradation oxydative de l'acide ascorbique. Une augmentation de l'acidité titrable de la pulpe a également été notée (+ $50 \%$ après 35 j) (résultats non illustrés). Ces évolutions pourraient être d'origine biochimique ; en effet, compte tenu de la faible teneur en eau du produit, il semble peu probable qu'elles soient liées à un développement microbien. Nous avons également constaté que la couleur de la pulpe fonçait pendant le stockage.

Les résultats obtenus montrent que d'importantes modifications de composition peuvent intervenir dans la pulpe du fruit de baobab pendant son stockage. Pour limiter la reprise en eau et les phénomènes oxydatifs, l'utilisation d'emballages étanches devra être envisagée.

\subsubsection{Composition des graines}

L'étude portant sur la pulpe du fruit a été complétée par une analyse de la composition des graines (tableau II). Celles-ci renferment environ $12 \%$ de lipides et $15 \%$ de protéines. Comme habituellement dans les graines, les sucres y sont peu représentés.

\subsection{Utilisations traditionnelles de la pulpe de fruit}

L'enquête réalisée a révélé que toutes les parties du baobab étaient utilisées : pulpe, graines, coque du fruit, feuilles, écorce, racines et même sève de la plante. 
Tableau II.

Principales caractéristiques des graines du fruit du baobab à partir d'échantillons collectés en 2006 sur un marché de Dakar (Sénégal). Les teneurs sont exprimées par rapport à la matière fraîche.

\begin{tabular}{lccccc}
$\begin{array}{l}\text { Humidité } \\
\left(\mathrm{g} \cdot 100 \mathrm{~g}^{-1}\right)\end{array}$ & $\begin{array}{c}\text { Acidité titrable } \\
\left(\mathrm{mEq} \cdot 100 \mathrm{~g}^{-1}\right)\end{array}$ & Sucres totaux & Sucres réducteurs & Protéines & Lipides \\
\hline 9,9 & 7 & 2,4 & 0,4 & $\left.100 \mathrm{~g}^{-1}\right)$ & Cendres \\
\hline
\end{tabular}

La pulpe du fruit, partie la plus fréquemment exploitée, est également la matière première quantitativement la plus consommée. Parmi ses cinq principales utilisations traditionnelles recensées, les boissons de type jus de fruit sont les plus couramment citées (figure 4). Le " ngalax " arrive en seconde position : ce mélange liquide sucré à base de pâte d'arachide, de pulpe de fruit du baobab et de farine de mil roulée cuite est surtout préparé à l'occasion de fêtes religieuses (Korité, Pâques). Enfin, la pulpe est également utilisée comme acidifiant dans la cuisine traditionnelle.

L'enquête menée a également permis de caractériser les procédés traditionnels d'élaboration du jus de fruit qui correspond en fait à un nectar (pulpe de fruit additionnée d'eau et de sucre). Le schéma technologique suivi est assez constant (figure 5). Quelques variantes ont cependant été relevées principalement lors des opérations de lavage (nombre de lavages, température de l'eau), d'extraction (durée du trempage et du malaxage, ratio [eau / pulpe] utilisé) et de la formulation (quantité de sucre, aromatisation). Les boissons obtenues sont de qualité inégale. Elles ne sont jamais stabilisées; cependant, elles peuvent être conservées à l'état réfrigéré pendant quelques jours.

\section{4. Étude de la transformation en nectar}

\subsection{1. Étude du procédé d'élaboration du nectar}

Faisant suite à l'enquête sur les utilisations traditionnelles de la pulpe du fruit de baobab, un procédé standard de transformation de la pulpe en nectar a été défini en choisissant les conditions de traitement les plus

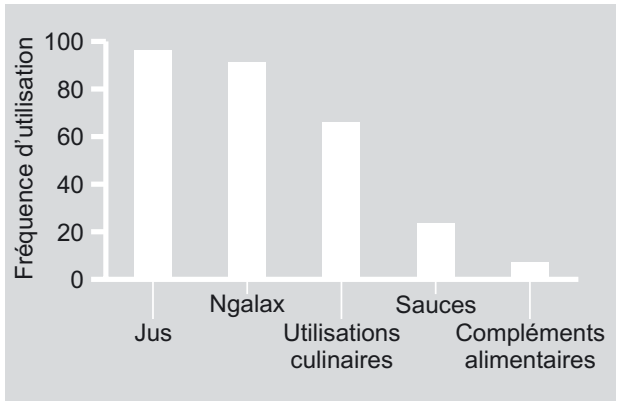

Figure 4.

Fréquence des principales utilisations alimentaires traditionnelles de la pulpe de fruit du baobab d'après une enquête réalisée sur un échantillon de 93 personnes interrogées à Dakar et à Saly (Sénégal). fréquemment citées au cours de l'enquête. La boisson obtenue correspond au nectar traditionnel " moyen " que nous appellerons nectar standard. Elle est préparée avec des rapports massiques [fruit / eau] de [1 / 3] et [fruit / sucre] de [1 / 0,8], le terme fruit correspondant au fruit décoqué. La séparation de la pulpe des graines et des fibres est réalisée par trempage, puis tamisage sur tamis de mailles à $0,5 \mathrm{~mm}$ de diamètre. Dans ces conditions, la boisson obtenue présente un extrait sec soluble voisin de $17 \mathrm{~g} \cdot 100 \mathrm{~g}^{-1}$, un $\mathrm{pH}$ de 3,3 et une acidité titrable d'environ $4 \mathrm{mEq} \cdot 100 \mathrm{~mL}^{-1}$.

Quelques unes des variantes identifiées au niveau des étapes de lavage et d'extraction ont été testées pour évaluer leur impact sur le rendement d'extraction et la qualité de la boisson finale (teneurs en solutés).

L'étape de lavage est nécessaire pour éliminer les impuretés souvent associées à la pulpe (sable, terre). Un examen visuel du produit (présence d'impuretés) montre que deux lavages successifs à température ambiante $\left(30^{\circ} \mathrm{C}\right)$ sont suffisants. Un seul lavage à l'eau chaude ou trois lavages à l'eau froide entraînent des pertes de matières sèches supérieures à $10 \%$.

Lors de l'étape d'extraction, nous avons mis en évidence la possibilité de réduire la 
Figure 5.

Procédé traditionnel

d'élaboration du nectar de fruit du baobab et principales variantes d'après une enquête réalisée sur un échantillon de 93 personnes (42 ménagères, 27 vendeuses de fruits, 18 restauratrices et 6 restaurateurs) dans la zone urbaine de Dakar et à Saly (Sénégal).

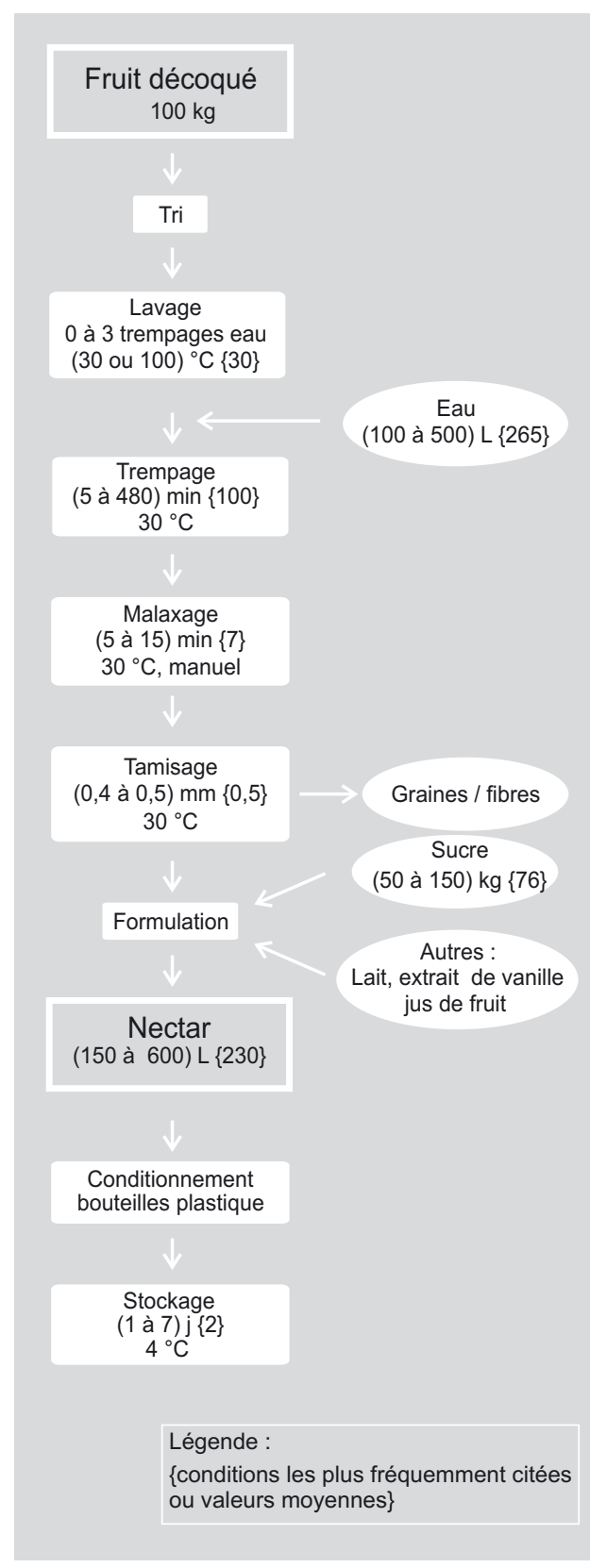

durée de traitement par rapport aux pratiques traditionnelles usuelles. En effet, la séparation de la pulpe des graines et des fibres peut être aisément obtenue en associant un trempage à froid de seulement $30 \mathrm{~min}$ à un malaxage de $4 \mathrm{~min}$. En terme sensoriel, un rapport massique [fruit / eau] de [1 / 3] est optimal. En effet, un ratio plus faible a conduit à une boisson jugée trop diluée par certains membres du jury d'évaluation sensorielle. Un ratio plus élevé aboutit à un produit jugé trop visqueux.

Plusieurs options ont été testées pour l'ajout de sucre dans le produit. Un nectar standard à $17^{\circ}$ Brix a été préparé avec un ratio [fruit / sucre] de $[1 / 0,8]$. Ce produit a été jugé trop sucré par certains membres du panel de dégustation. En conséquence, un nectar à $13{ }^{\circ}$ Brix avec un ratio [fruit / sucre] de $[1 / 0,5]$ a également été préparé. Pour certains autres essais, le nectar n'a pas été sucré.

\subsubsection{Caractéristiques des nectars obtenus}

Les principales caractéristiques des différents nectars obtenus en adoptant la procédure de préparation optimisée ont été comparées.

En utilisant un ratio [fruit / eau] de [1 / 3] lors de l'étape d'extraction, le pH des boissons obtenues a varié entre 3,1 et 3,5 (tableau III). Comparativement à d'autre jus de fruits, le produit est donc assez acide. Ces valeurs de $\mathrm{pH}$ sont surprenantes au regard des faibles acidités titrables mesurées [(4 à 7) $\left.\mathrm{mEq} \cdot 100 \mathrm{~mL}^{-1}\right]$. Les teneurs en acide ascorbique ont oscillé entre (12 et 14) $\mathrm{mg} \cdot 100 \mathrm{~mL}^{-1}$. Les nectars contiennent environ $0,2 \%$ de minéraux et $0,15 \%$ de protéines. Sans ajout de sucre, l'extrait sec soluble du nectar a avoisiné les $7 \mathrm{~g} \cdot 100 \mathrm{~g}^{-1}$.

Afin d'évaluer l'impact de la composition du rapport massique [fruit / eau], d'autres nectars non sucrés ont été préparés en utilisant davantage d'eau lors de l'étape d'extraction. Les nectars préparés avec un ratio [fruit / eau] de [1 / 4] et [1 / 5] ont présenté des extraits secs solubles de $5 \mathrm{~g} \cdot 100 \mathrm{~g}^{-1}$ et $4 \mathrm{~g} \cdot 100 \mathrm{~g}^{-1}$, respectivement. L'acidité titrable et la teneur en acide ascorbique ont diminué logiquement dans un rapport voisin des extraits secs solubles. Le pH n'a pas été significativement affecté par la dilution, ce qui s'explique par le pouvoir tampon du produit.

Dans tous les cas, les boissons obtenues ne se sont conservées que $4 \mathrm{j}$ à $4{ }^{\circ} \mathrm{C}$ car, audelà, des modifications significatives de couleur et de flaveur y ont été détectées. 


\begin{tabular}{|c|c|c|c|c|c|c|c|c|c|}
\hline \multirow[t]{2}{*}{$\begin{array}{l}\text { Type de } \\
\text { nectar }\end{array}$} & \multirow[t]{2}{*}{$\begin{array}{l}\text { Composition du } \\
\text { nectar }\end{array}$} & $\begin{array}{l}\text { Matière } \\
\text { sèche }\end{array}$ & $\begin{array}{l}\text { Extrait sec } \\
\text { soluble }\end{array}$ & Saccharose & \multirow[t]{2}{*}{$\begin{array}{l}\text { Acidité titrable } \\
\left(\mathrm{mEq} \cdot 100 \mathrm{~mL}^{-1}\right)\end{array}$} & \multirow[t]{2}{*}{$\mathrm{pH}$} & Cendres & Protéines & \multirow{2}{*}{$\begin{array}{c}\text { Acide } \\
\text { ascorbique } \\
\left(\mathrm{mg} \cdot 100 \mathrm{~mL}^{-1}\right)\end{array}$} \\
\hline & & \multicolumn{3}{|c|}{$\left(g \cdot 100 g^{-1}\right)$} & & & \multicolumn{2}{|c|}{$\left(g \cdot 100 g^{-1}\right)$} & \\
\hline \multirow[t]{2}{*}{ Nectar sucré } & $\begin{array}{c}\text { Fruit / eau / sucre } \\
{[1 / 3 / 0,8]}\end{array}$ & 24,2 & 17 & 14,2 & 4,0 & 3,32 & 0,30 & 0,15 & 12,1 \\
\hline & $\begin{array}{c}\text { Fruit / eau / sucre } \\
{[1 / 3 / 0,5]}\end{array}$ & 14,6 & 12,6 & 11 & 3,8 & 3,32 & 0,20 & 0,15 & 13,2 \\
\hline \multirow[t]{3}{*}{$\begin{array}{l}\text { Nectar non } \\
\text { sucré }\end{array}$} & $\begin{array}{c}\text { Fruit / eau } \\
{[1 / 3]}\end{array}$ & 7,6 & 6,8 & - & $5,3-6,8$ & $3,06-3,51$ & 0,15 & 0,14 & $12,9-14,1$ \\
\hline & $\begin{array}{c}\text { Fruit / eau } \\
{[1 / 4]}\end{array}$ & 5,7 & 5,0 & - & 4,8 & 3,01 & 0,15 & 0,14 & 11,3 \\
\hline & $\begin{array}{l}\text { Fruit / eau } \\
\quad[1 / 5]\end{array}$ & 4,9 & $3,5-4,0$ & - & $4,1-4,8$ & $3,03-3,50$ & 0,15 & 0,15 & $7,5-9,4$ \\
\hline
\end{tabular}

\section{Tableau IV.}

Impact de la pasteurisation sur la composition des nectars de fruit ([fruit / eau] = [1 / 3]) obtenus à partir de la transformation de la pulpe du fruit du baobab (Sénégal).

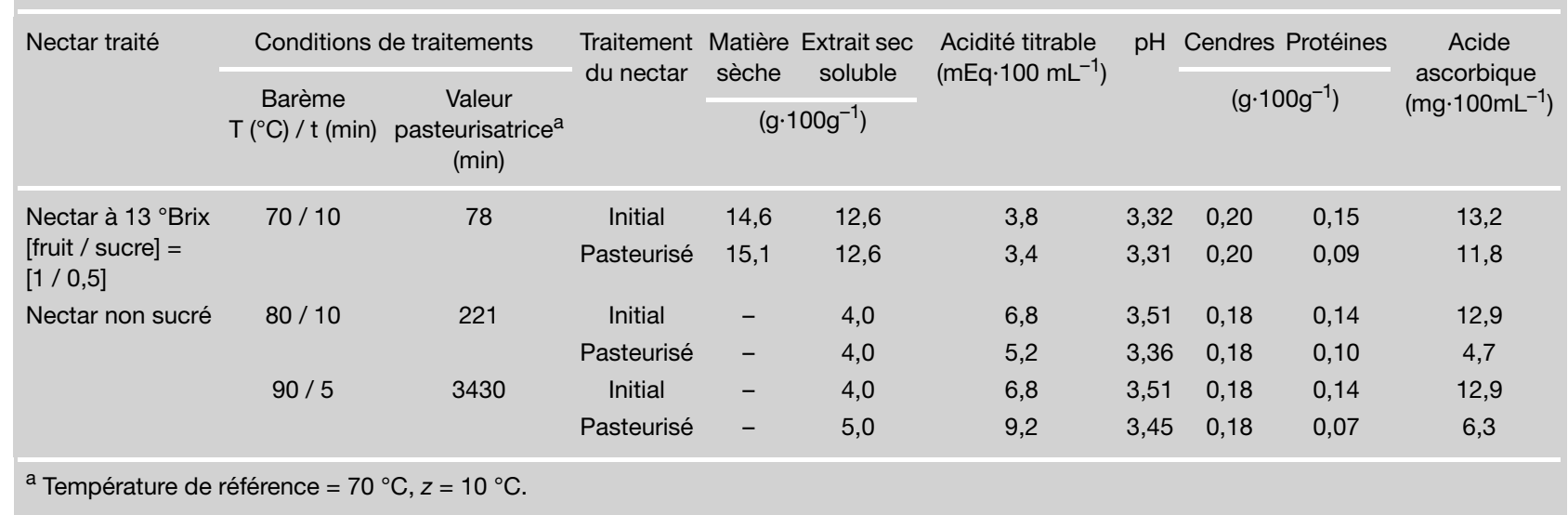

\subsection{Stabilisation des nectars par pasteurisation thermique}

\subsubsection{Efficacité du traitement}

L'évaluation de l'efficacité du traitement thermique a été réalisée à partir de dénombrements microbiologiques et de tests de stabilité.

En utilisant un barème à $70{ }^{\circ} \mathrm{C}$ pendant $10 \mathrm{~min}$, la valeur pasteurisatrice subie par le produit a été de près de 80 min pour un nectar à $13^{\circ}$ Brix (tableau IV).
Les résultats des analyses microbiologiques ont montré que la pulpe du fruit était relativement peu contaminée avec une flore aérobie totale de l'ordre de $2,5 \times 10^{3} \mathrm{UFC} \cdot \mathrm{g}^{-1}$ (tableau $\mathrm{V}$ ). Cette flore est constituée essentiellement de moisissures et de bactéries lactiques.

Les résultats obtenus sur le nectar préparé à partir de la pulpe ont conduit à mettre en évidence des contaminations liées aux manipulations et au matériel. En terme microbiologique, l'efficacité du traitement thermique utilisé semble démontrée et le 
Tableau V.

Dénombrements microbiologiques dans la pulpe et dans le nectar obtenus à partir de la transformation du fruit du baobab (Sénégal).

\begin{tabular}{lccc} 
Analyses & $\begin{array}{c}\text { Pulpe } \\
\left(\mathrm{UFC} \cdot \mathrm{g}^{-1}\right)\end{array}$ & $\begin{array}{c}\text { Nectar à } 13^{\circ} \text { Brix } \\
\left(\mathrm{UFC} \cdot \mathrm{mL}^{-1}\right)\end{array}$ \\
& & Initial & Pasteurisé à $70^{\circ} \mathrm{C}$ \\
\hline $\begin{array}{l}\text { Flore mésophile aérobie totale } \\
\text { Bactéries lactiques }\end{array}$ & $1,2-3,7 \times 10^{3}$ & $3,1 \times 10^{2}-5,6 \times 10^{5}$ & $<10^{2}$ \\
Levures & $5,0 \times 10^{2}-2,6 \times 0^{3}$ & $1,6 \times 10^{2}-1,3 \times 10^{4}$ & Absent dans $1 \mathrm{~mL}$ \\
Moisissures & $<10$ & $<10$ & Absent dans $0,1 \mathrm{~mL}$ \\
Salmonelles & $4,0 \times 10^{2}-2,0 \times 10^{3}$ & $2 \times 10^{1}-3,4 \times 10^{3}$ & $<10^{2}$ \\
Coliformes & Absent dans $25 \mathrm{~g}$ & Absent dans $25 \mathrm{~mL}$ & Absent dans $25 \mathrm{~mL}$
\end{tabular}

produit obtenu est conforme aux normes en vigueur pour les nectars pasteurisés. Des tests de conservation ont été réalisés en complément. Malgré les bons résultats des analyses microbiologiques, des modifications de flaveur du nectar pasteurisé ont été détectées après $11 \mathrm{j}$ de stockage à $4{ }^{\circ} \mathrm{C}$. Ces modifications sensorielles ont été associées à d'importantes modifications de la composition du produit : diminution de la teneur en sucre totaux de (11 à 7) $\mathrm{g} \cdot 100 \mathrm{~g}^{-1}$ $(-35 \%)$ et augmentation de la teneur en éthanol de $(0,9$ à 1,4$) \% \mathrm{vol}(+50 \%)$. En utilisant les conditions de traitement que nous avons testées, la stabilisation du produit ne semble donc pas effective.

Du fait de ces résultats, des barèmes plus élevés ont été testés sur nectar non sucré : $80{ }^{\circ} \mathrm{C}$ pendant $10 \mathrm{~min}$ et $90{ }^{\circ} \mathrm{C}$ pendant $5 \mathrm{~min}$. Ces traitements ont conduits à des valeurs pasteurisatrices de (221 et 3430) min, respectivement (tableau $I V$ ). Des tests de conservation en étuve à $37^{\circ} \mathrm{C}$ (vieillissement accéléré) pendant 8 j ont été menés afin d'évaluer la stabilité des nectars pasteurisés. Le suivi au cours du temps des caractéristiques des produits a montré que les variations du $\mathrm{pH}$, de l'acidité titrable, de l'extrait sec soluble et de la teneur en acide ascorbique étaient inférieures à $10 \%$. Il n'y a donc pas alors d'évolution importante de la composition du produit. La stabilisation du nectar est donc atteinte, contrairement au traitement précédent effectué à $70{ }^{\circ} \mathrm{C}$. Afin de confirmer ces résultats, il sera néanmoins nécessaire de réaliser des tests de conservation sur plusieurs semaines.
Notre travail a prouvé qu'il était possible de stabiliser le nectar de pulpe de fruits du baobab via un traitement thermique adapté. Ces études devront être complétées par la recherche de la valeur pasteurisatrice minimale nécessaire, vraisemblablement comprise entre (80 et 220) min.

\subsubsection{Impact du traitement sur la qualité du nectar}

La comparaison de la composition des nectars avant et après pasteurisation a montré que, pour le traitement à $70{ }^{\circ} \mathrm{C}$, la composition du produit était peu modifiée par la pasteurisation malgré une perte de $10 \%$ notée pour le taux de vitamine C (tableau IV). Les résultats obtenus ont montré que l'odeur et la couleur du nectar étaient altérées par le traitement. En revanche, aucune différence significative n'a été mise en évidence pour les autres descripteurs (Student $p<0,05$ ).

Les traitements à (80 et 90$){ }^{\circ} \mathrm{C}$ ont conduit à des pertes beaucoup plus importantes de vitamine C. En effet les teneurs mesurées sur les nectars pasteurisés ont été de (50 à 60)\% plus faibles que celles mesurées sur le nectar initial. La qualité nutritionnelle de la boisson a donc été fortement dégradée en termes de teneur en vitamines. Bien qu'il n'ait pas été évalué au cours de cette étude, l'impact de ces traitements à (80 et 90$){ }^{\circ} \mathrm{C}$ sur la qualité sensorielle du produit doit être, vraisemblablement, plus marqué qu'à $70{ }^{\circ} \mathrm{C}$.

Dans les conditions de traitement choisies, la stabilité du nectar pourrait donc être 


\begin{tabular}{|c|c|c|c|c|c|c|}
\hline \multirow[t]{2}{*}{ Pilote } & \multirow{2}{*}{$\begin{array}{l}\text { Vitesse tangentielle } \\
\text { de circulation } \\
\left(\mathrm{m} \cdot \mathrm{s}^{-1}\right)\end{array}$} & \multirow[t]{2}{*}{$\begin{array}{c}\text { Température } \\
\left({ }^{\circ} \mathrm{C}\right)\end{array}$} & \multirow{2}{*}{$\begin{array}{c}\text { Pression } \\
\text { transmembranaire } \\
\text { (bar) }\end{array}$} & \multirow{2}{*}{$\begin{array}{l}\text { Facteur de } \\
\text { réduction } \\
\text { volumique }\end{array}$} & \multicolumn{2}{|c|}{$\begin{array}{l}\text { Densité de flux de perméat } \\
\left(L \cdot h^{-1} \cdot \mathrm{m}^{-2}\right)\end{array}$} \\
\hline & & & & & $\begin{array}{l}\text { Pour le nectar } \\
\quad[1 / 3]\end{array}$ & $\begin{array}{c}\text { Pour le nectar } \\
{[1 / 5]}\end{array}$ \\
\hline \multirow{4}{*}{$\begin{array}{l}\text { Semi-industriel } \\
\text { IMECA }\end{array}$} & 2,0 & 30 & 1,00 & 2,1 & $25^{1}$ & - \\
\hline & & & 2,25 & 1,8 & - & - \\
\hline & & & 2,85 & 1,4 & - & - \\
\hline & 4,4 & 41 & 2,80 & 1,9 & 45 & - \\
\hline \multirow[t]{8}{*}{ De laboratoire TIA } & 6,0 & 25 & 2,25 & 2,0 & 30 & - \\
\hline & & & 2,75 & - & 26 & - \\
\hline & & & 3,25 & - & 29 & - \\
\hline & & & 3,75 & - & 23 & - \\
\hline & & & 2,25 & 1,0 & - & 77 \\
\hline & & & 2,75 & - & - & 51 \\
\hline & & & 3,25 & - & - & 39 \\
\hline & & & 3,75 & - & - & 32 \\
\hline
\end{tabular}

obtenue, mais cela au détriment de la qualité du produit. Afin de limiter les dégradations thermiques, une étude complète visant l'optimisation du barème de pasteurisation sera nécessaire afin d'atteindre la valeur pasteurisatrice minimale permettant de stabiliser le nectar.

\subsection{Stabilisation par microfiltration tangentielle}

\subsubsection{Performances de la filtration}

Les valeurs de densités de flux mesurées après stabilisation, après (30 à 100) min de filtration selon les cas, ont révélé que, pour le nectar préparé avec un ratio [fruit eau $]=[1 / 3]$, les densités de flux étaient comprises entre (23 et 45$) \mathrm{L} \cdot \mathrm{h}^{-1} \cdot \mathrm{m}^{-2}$ pour des facteurs de réduction volumique (FRV) compris entre 1,4 et 2,1 selon l'installation considérée (tableau VI). Quelle que soit l'installation utilisée, la densité de flux n'a pas significativement dépendu de la pression transmembranaire.

Sur le pilote IMECA, l'augmentation de la vitesse de circulation [de $(2,0$ à 4,4$) \mathrm{m} \cdot \mathrm{s}^{-1}$ ] et de la température [(de 30 à 41$\left.)^{\circ} \mathrm{C}\right]$ a amélioré les flux qui sont passés de (25 à
45) $\mathrm{L} \cdot \mathrm{h}^{-1} \cdot \mathrm{m}^{-2}$. Néanmoins, comparativement à d'autre jus de fruit, tels que l'orange, le melon ou le fruit de la passion, ces valeurs sont restées faibles [24-26]. Ces résultats seraient principalement liés à la viscosité élevée du produit qui est passé de $6 \mathrm{mPa} \cdot \mathrm{s}^{-1}$ en début de filtration à environ $60 \mathrm{mPa} \cdot \mathrm{s}^{-1}$ à $\mathrm{FRV}=2$. Dans les conditions testées et en partant d'un nectar à [1 / 3], les performances de la filtration ont donc été médiocres. Elles se révèlent insuffisantes pour envisager une application industrielle. Il est probable que le couplage du procédé avec une liquéfaction enzymatique partielle permettrait d'améliorer ces résultats [26, 27]. Néanmoins, cette option n'a pas été retenue compte tenu du surcoût qu'engendrerait la consommation d'enzymes.

Des essais complémentaires ont été réalisés sur un nectar préparé avec un ratio [fruit / eau $]=[1 / 5]$ (pilote TIA de laboratoire) . Les densités de flux obtenues à FRV $=1$ ont été comprises entre (32 et 77) $\mathrm{L}^{\circ} \mathrm{h}^{-1} \cdot \mathrm{m}^{-2}$ (figure O). La viscosité moins élevée du produit et le FRV plus faible utilisé expliqueraient cette amélioration. Contrairement au cas précédent, la pression transmembranaire a influé sur la densité de flux. Les meilleurs résultats ont été obtenus aux plus faibles pressions. 
Figure 6.

Évolution des densités de flux de perméat lors de la microfiltration tangentielle d'un nectar (rapport massique [fruit / eau $]=[1 / 5])$ de fruit de baobab à différentes pressions transmembranaires.

Température $=25^{\circ} \mathrm{C}$; vitesse tangentielle de circulation $=$ $6 \mathrm{~m} \cdot \mathrm{s}^{-1}$; facteur de réduction volumique $=1$ (pilote $\mathrm{TIA}$ laboratoire).

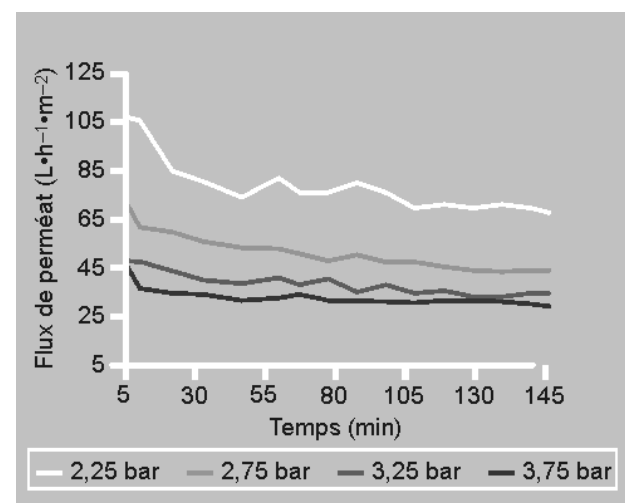

Bien que ces résultats soient encourageants, il ne nous paraît pas judicieux de poursuivre dans cette voie car le nectar [1/5], trop dilué, ne présente pas une qualité sensorielle satisfaisante.

\subsubsection{Qualité des produits}

Les compositions en solutés des différents rétentats et perméats obtenus ont été comparées à celle des nectars bruts (tableau VII) Dans tous les cas, les perméats ont été limpides et parfaitement clarifiés. Leur viscosité a été comprise entre (1 et 2$) \mathrm{mPa} \cdot \mathrm{s}^{-1}$.

Quel qu'ait été l'essai, l'extrait sec soluble a augmenté dans le rétentat et diminué dans le perméat par rapport au nectar initial. Cela indiquerait qu'une partie des solutés a été retenue par la membrane. Il pourrait s'agir de macromolécules solubles car les sucres ne peuvent être retenus sur ce type de membrane. De plus, la présence de pulpe dans le produit pourrait interférer sur les mesures d'indice réfractométrique [25, 27].

Sur le pilote IMECA, l'acidité titrable a été voisine dans les trois fractions de nectar. En revanche, sur le pilote TIA, l'acidité a été plus élevée dans le rétentat et plus faible dans le perméat. Ce résultat est surprenant, la masse molaire des acides organiques présents étant trop faible pour qu'ils soient retenus.

Enfin, en ce qui concerne la vitamine C, sur le pilote semi-industriel (IMECA), le perméat a subi une perte de (20 à 30) \%, alors que, sur le pilote de laboratoire, les pertes ont été nettement plus importantes. Ces pertes observées aussi bien dans le perméat que dans le rétentat pourraient résulter d'une dégradation oxydative liée à l'aération des produits. Quelques précautions de manipulation pour éviter l'incorporation d'oxygène dans les jus devraient permettre de les limiter.

L'évaluation sensorielle d'un nectar reconstitué à partir de perméat et de rétentat a montré que la couleur et le goût (sucré, acide) du produit étaient peu différents de ceux du nectar frais. En revanche, l'odeur et le caractère pulpeux ont semblé significativement atténués par le traitement. Des tests comparatifs devront être entrepris après avoir optimisé le barème de pasteurisation du rétentat afin de mieux évaluer le gain qualitatif probable des nectars microfiltrés par rapport aux nectars pasteurisés.

\section{Conclusion}

L'analyse des échantillons collectés dans différentes zones géographiques du Sénégal a montré que la pulpe du fruit de baobab se caractérisait par une faible teneur en eau, une forte acidité et des teneurs élevées en sucres, acide ascorbique, minéraux et amidon. Les teneurs en acide ascorbique et autres acides organiques ont été plus variables. Le pouvoir antioxydant élevé de la pulpe pourrait être lié à sa richesse en acide ascorbique et en composés phénoliques. Huit des dix composés d'arôme décelés dans cette pulpe ont été identifiés. Trois composés prédominent : un ester, un composé phénolique et un aldéhyde. Les résultats que nous avons obtenus sur une large gamme de matière première confirment et complètent les valeurs présentées dans la littérature. Néanmoins, la caractérisation biochimique du fruit mériterait d'être approfondie par une étude détaillée de la fraction polyosidique (pectines, mucilages), des composés phénoliques, des vitamines hydrosolubles du groupe B et des caroténoïdes. Un travail complémentaire sera également nécessaire pour mieux caractériser la composition aromatique du produit.

D'après nos résultats, sans précaution particulière, la composition de la pulpe évoluerait au cours du stockage et sa qualité se dégraderait rapidement. La pulpe sèche 
Transformation du fruit du baobab




étant la forme de commercialisation du fruit la plus répandue, une étude spécifiquement dédiée à sa conservation serait à entreprendre. Ce travail permettrait de mieux comprendre les mécanismes responsables des dégradations et de définir les conditions de conservation les mieux adaptées au produit.

L'enquête réalisée sur les voies de valorisations traditionnelles a mis en évidence que la pulpe du fruit de baobab était principalement utilisée pour l'élaboration de boissons de type nectar. Ces boissons sont largement consommées au niveau local. Le schéma de fabrication suivi est relativement constant. La séparation de la pulpe des graines est réalisée par voie humide. Les boissons obtenues sont de qualité inégale et ne sont jamais stabilisées. En se basant sur les résultats de cette enquête, un procédé d'élaboration de référence a tout d'abord été défini. Des améliorations portant sur les étapes de lavage et d'extraction ont ensuite été proposées pour obtenir, avec un rendement en matière maximal, une boisson de qualité satisfaisante et pour diminuer la durée totale du traitement.

Bien que la matière première soit peu contaminée (flore essentiellement constituée de moisissures et de bactéries lactiques), un barème de pasteurisation de $70{ }^{\circ} \mathrm{C}$ pendant $10 \mathrm{~min}$ (valeur pasteurisatrice de $80 \mathrm{~min}$ ) s'est révélé insuffisant pour stabiliser le nectar. La stabilisation pourrait néanmoins être obtenue en utilisant des barèmes plus élevés $\left(80^{\circ} \mathrm{C}\right.$ pendant $10 \mathrm{~min}$, valeur pasteurisatrice de $221 \mathrm{~min}$ ). Cependant, la qualité du produit est alors significativement altérée par le traitement, aussi bien au niveau sensoriel que vitaminique. Afin de limiter ces dégradations tout en garantissant l'efficacité du traitement, il sera nécessaire d'envisager une optimisation du barème.

L'intérêt de la microfiltration tangentielle pour la stabilisation à froid du nectar de pulpe de fruit de baobab apparaît compromis par les faibles densités de flux de perméat mesurées. Bien que les performances du procédé puissent être améliorées en associant un traitement enzymatique à la filtration et que les caractéristiques de la matière première semblent ainsi mieux préservées, cette voie technologique ne nous parait pas adaptée pour le traitement du produit.
À l'avenir, dans la perspective d'une production locale de nectar de fruit du baobab, les travaux de recherche devront être focalisés sur la stabilisation thermique de la boisson. Enfin, il sera intéressant de travailler également sur la production de pulpe en poudre séparée simplement des graines par voie sèche. À condition qu'elle soit de bonne qualité et qu'elle puisse se conserver sans difficulté, cette poudre pourrait être directement commercialisée (éventuellement additionnée de sucre) et utilisée pour reconstituer une boisson après solubilisation dans l'eau.

\section{Remerciements}

Ce travail s'intègre dans le cadre du projet "Valorisation du fruit du baobab " (partenariat ESP / Montpellier SupAgro / CIRAD) qui a bénéficié d'un support financier du réseau des chercheurs en Génie des Procédés Appliqués à l'Agro-alimentaire (GP3A) de l'Agence Universitaire de la Francophonie (AUF). Les auteurs remercient Aïda Gabar Diop, Mame Saye Fall, Bénédicte Diatta, Mouhamadou Lamine Ndong et Amath Thiema pour leur participation à ce travail.

\section{Références}

[1] Berhaut J., Flore illustrée du Sénégal, tome 2, Clairafrique, Dakar, Sénégal, 1974.

[2] Haddad C., Fruitiers sauvages du Sénégal, Univ. Montpellier I, Thèse, Montpellier, France, 2000.

[3] Wickens G.E., The baobab-Africa's upsidedown tree, Kew Bull. 37 (1982) 173-209.

[4] Diop A.G., Sakho M., Dornier M., Cisse M., Reynes M., Le baobab africain (Adansonia digitata L.) : principales caractéristiques et utilisations, Fruits 61 (2005) 55-69.

[5] Guinko S., Rôle de quelques espèces ligneuses spontanées pour les programmes d'amélioration génétique et de reforestation, in: Sémin. Rég. Semen. For., Ouagadougou, Burkina Faso, 1988.

[6] Ralaimanarivo A., Contribution à l'étude analytique de six espèces de baobab : application de l'étude de la fraction grasse à leur chimiotaxonomie, Fac. Sci. Tech., Univ. Droit Écon. Sci., Thèse, Aix-Marseille, France, 1980. 
[7] Codjia J.T.C., Fonton B.K., Assogbadjo A., Ekue M.R.M., Le baobab, une espèce à usage multiple au Bénin, Cent. Int. d'Écodév. Intégr. (CECODI), Cotonou, Bénin, 2001.

[8] Fall T., Étude sur la collecte et l'analyse des données sur les produits forestiers non ligneux au Sénégal, FAO, For. Stat. Data Coll., Rome, Italie, 2001.

[9] Obizoba I.C., Anyika J.U., Nutritive value of baobab milk (gubdi) and mixtures of baobab (Adansonia digitata L.) and hungry rice, acha (Digitaria exilis) flours, Plant Foods Hum. Nutr. 46 (1994) 157-165.

[10] Saka J., Rapp I., Akinnifesi F., Ndolo V., Mhango J., Physicochemical and organoleptic characteristics of Uapaca kirkiania, Strychnos cocculoides, Adansonia digitata and Mangiferia indica fruit products, Int. J. Food Sci. Technol. 42 (2007) 836-841.

[11] Favier J.C., Ireland-Rippert J., Laussucq C., Feinberg M., Répertoire général des aliments, tome 3, Table de composition des fruits exotiques, fruits de cueillette d'Afrique, Lavoisier, Paris, France, 1993.

[12] Soloviev P., Niang T.D., Gaye A., Totte A., Variabilité des caractères physico-chimiques des fruits de trois espèces ligneuses de cueillette, récoltés au Sénégal : Adansonia digitata, Balanites aegytiaca et Tamarindus indica, Fruits 59 (2004) 109-119.

[13] Anon., Fruits and fruits products, in: Helrich K. (Ed.), Official methods of analysis of the association of official analytical chemists (AOAC), Arlington, USA, 1990, pp. 910-928.

[14] Anon., Jus de fruits et de légumes. Spécifications et méthodes d'analyse, $2 \mathrm{e}$ éd., Afnor, Paris, France, 1996.

[15] Georgé S., Brat P., Alter P., Amiot M.J., Rapid determination of polyphenols and vitamin $\mathrm{C}$ in plant-derived products, J. Agric. Food Chem. 53 (2005) 1370-1373.

[16] Vaillant F., Perez A., Davila I., Dornier M., Reynes M., Colorant and antioxidant properties of red-purple pitahaya (Hylocereus sp.), Fruits 60 (2005) 3-12.

[17] Brat P., Yahia A., Chillet M., Bugaud C., Bakry M., Reynes M., Brillouet J.M., Influence of cultivar, growth altitude, and maturity stage on banana volatile compound composition, Fruits 59 (2004) 75-82.

[18] Guiraud J.P., Microbiologie alimentaire, Dunod, Paris, 1998.

[19] Murray S.S., Schoeninger M.J., Bunn H.T., Pickering T.R., Marlet J.A., Nutritional composition of some wild plant foods and honey used by Hadza foragers of Tanzania, J. Food Compos. Anal. 14 (2001) 3-13.

[20] Bourgeois C.F., Les vitamines dans les industries agroalimentaires, Tec \& Doc., Coll. Sci. Tech. Agroaliment., Paris, France, 2002.

[21] Rodrigues R., De Menezes H., Cabral L., Dornier M., Reynes M., An amazonian fruit with a high potential as natural source of vitamin C: the camu-camu (Myrciaria dubia), Fruits 56 (2001) 345-354.

[22] Xu B.J., Yuan S.H., Chang, S.K.C., Comparative analyses of phenolic composition, antioxidant capacity, and color of cool season legumes and other selected food legumes, J. Food Sci. 72 (2007) 167-177.

[23] Lam H., Proctor A., Howard L., Cho M.J., Rapid fruit extracts antioxidant capacity determination by Fourier transform infrared spectroscopy, J. Food Sci. 70 (2005) 545549.

[24] Cisse M., Vaillant F., Dornier M., Perez A.M., Reynes M., Quality of orange juice processed by coupling crossflow microfiltration and osmotic evaporation, Int. J. Food Sci. Technol. 40 (2005) 105-116.

[25] Vaillant F., Cisse M., Dornier M., Perez A.M., Reynes, M., Clarification and concentration of melon juice on an industrial pilot scale using crossflow microfiltration and osmotic evaporation, J. Innov. Food Sci. Emerg. Technol. 6 (2005) 213-220.

[26] Vaillant F., Millan P., O'Brien G., Dornier M., Decloux M., Reynes M., Crossflow microfiltration of passion fruit juice after partial enzymatic liquefaction, J. Food Eng. 42 (1999) 215-224.

[27] Vaillant F., Dornier M., Decloux M., Reynes M., Strategy for economical optimisation of the clarification of pulpy fruit juices using crossflow microfiltration. J. Food Eng. 48 (2001) 83-90. 


\section{Caracterización de los frutos del baobab y estudio de su transformación en néctar.}

Resumen - Introducción. El baobab (Adansonia digitata L.) es un árbol que crece de forma silvestre en Senegal, donde el fruto se consume bajo diversas formas. Aunque la ecología y la botánica de la planta han sido bien estudiadas, existen pocos trabajos sobre el procesamiento de la fruta o el impacto del tratamiento sobre la calidad de los productos terminados. Para evaluar el potencial tecnológico de estas frutas, antes de poder desarrollar el mercado local, regional e internacional de los productos transformados, hemos tratado de caracterizar las materias primas (desde el punto de vista de su composición bioquímica y calidad nutricional), y estudiar la transformación del producto como néctar. Materiales y métodos. El estudio se centró sobre doce muestras de frutas de Adansonia digitata L. cosechadas entre 2004 y 2006 en diferentes zonas de Senegal. La proporción de los diversos componentes de las frutas (pulpa, semillas, fibras) se determinó, además de analizar las diferentes características y propiedades de la pulpa y de las semillas. Se estudió a flora microbiológica de las bebidas preparadas tradicionalmente. Se aplicaron también las técnicas de pasteurización y microfiltración tangencial a los néctares. Los diversos usos y el proceso tradicional de las frutas fueron evaluados a partir de una encuesta realizada sobre una muestra de 93 personas entrevistadas en las regiones de Dakar y Saly en Senegal. Resultados y discusión. La pulpa se caracteriza por un bajo contenido de agua (6-15\%), una alta acidez (68-201 mEq $100 \mathrm{~g}^{-1}$ ) y altos niveles de azúcares (20-32\%), ácido ascórbico (125-312 mg·100 g $\mathrm{g}^{-1}$ ), minerales (6\%) y almidón (41-47\%). Los cromatogramas obtenidos por cromatografía de gases acoplada a espectrometría de masas de la pulpa revelaron 10 compuestos volátiles como el miristato de isopropilo, un compuesto fenólico no identificado y el nonanal. Sin precauciones en particular la composición de la pulpa cambió durante el almacenamiento $\left(30^{\circ} \mathrm{C}\right)$ y su calidad se deterioró rápidamente. La encuesta mostró que la pulpa del fruto de baobab ha sido utilizada principalmente para la elaboración de bebidas tipo néctar en proporciones de masa [frutas : agua] de [1:3]. La estabilización de néctar se puede obtener por pasteurización $\left(80^{\circ} \mathrm{C} / 10 \mathrm{~min}\right.$ ) a expensas de la calidad de los productos. Se realizaron pruebas de estabilización del néctar por microfiltración tangencial empleando una proporción másica [frutas y agua] de [1:3] y [1: 5]. La densidad de flujo obtenida osciló entre $\left(23\right.$ y 77) $\mathrm{L} \cdot \mathrm{h}^{-1} \cdot \mathrm{m}^{-2}$ con factores de reducción de volumen (FRV) entre 1 y 2 . La baja densidad de flujos de permeado alcanzados limitarían el interés por aplicar la microfiltración tangencial para estabilizar en frío los néctares. Conclusión. En un futuro, con una perspectiva de producción local de néctar a partir del fruto de baobab, la investigación debe centrarse en la estabilización térmica de la bebida.

\section{Senegal / Adansonia / jugo de frutas / aptitud para la conservación / pasteurización / microfiltración}

